\title{
Aerobic exercise training prevents impairment in renal parameters and in body composition of rats fed a high sucrose diet
}

\author{
Jaqueline A. de Souza ${ }^{3 \dagger}$, Angélica B. Gonçalves Pinto ${ }^{1 \dagger}$, Emerson C. de Oliveira', Daniel B. Coelho ${ }^{1}$, \\ Nádia L. Totou ${ }^{2}$, Wanderson G. de Lima² and Lenice K. Becker ${ }^{1 *} \mathbb{C}$
}

\begin{abstract}
Objective: This study aimed to evaluate the effect of swimming training $(T)$ on the renal system and body composition parameters in young animals treated with a high sucrose diet (SUD) during 12 weeks.

Results: The SUD impaired the physical performance, increased the body adiposity index (BAI), Lee index (LI) and retroperitoneal adipose tissue (RAT) weight, plasma creatinine and number renal cells nuclei, decreased urinary volume and urinary creatinine excretion besides creatinine clearance. The T reversed the increased the BAI, LI, RAT weight, plasma and urinary creatinine, creatinine clearance and number renal cells nuclei in addition to promoting decrease in urinary protein excretion. This study found that eight weeks of swimming physical training protected renal function and restored normal glomerular filtration rate (GFR) values. Swimming training also contributed to prevention of the onset of a renal inflammatory process and caused a decrease in the risk of development of obesity promoted by SUD decreasing the body composition parameters (BAl, LI, and RAT weight).
\end{abstract}

Keywords: SUD, Swimming, Renal function, Body composition

\section{Introduction}

A high sugar level is a factor that has been linked to an increase in kidney disease and association with some risk factors, including diabetes, gout, metabolic syndrome [14] and the development of obesity. Besides an increase in oxidative stress and nitric oxide inactivation via reactive oxygen species, high sugar also induces high level of creatinine and blood urea nitrogen [5]. Sucrose, specifically, can promote an increase in proteinuria and a higher percentage of abnormal glomeruli in obese rats [6].

\footnotetext{
*Correspondence: lenice@ufop.edu.br

†Jaqueline Aparecida de Souza and Angélica Barbosa Gonçalves Pinto contributed equally to the work

${ }^{1}$ Physical Education School, Federal University of Ouro Preto (UFOP),

Ouro Preto, MG 35,400-000, Brazil

Full list of author information is available at the end of the article
}

Physical exercise is recognized to promote alterations in renal parameters in experimental animal models [7-9] those alterations provide renal protective effects. The exercise training neutralize the accumulation of renal triglycerides associated with high sucrose diet, which is partially mediated by upregulation of fatty acid $\beta$-oxidation [7].

Data have shown that a metabolic re-programming process subsequent to early-life insults, i.e. maternal life or childhood can take to development of chronic diseases $[10,11]$. The use of food rich in refined sugar has been shown to exponentially raise glucose blood levels, increase the total caloric intake and the prevalence of metabolic syndrome in children and adolescents these populations [12].

A balanced diet and regular physical exercise are of great relevance in preventing obesity, cardiovascular and kidney diseases, justifying the development of studies 
that assess the beneficial effects of regular physical training in childhood that will contribute to the decrease in deleterious effects of consuming a diet rich in simple carbohydrates.

Thus, this study aimed to evaluate the effect of swimming training $(\mathrm{T})$ on the renal system and body composition parameters [body adiposity and Lee indices (BAI and LI, respectively) and retroperitoneal adipose tissue (RAT) weight] in animals treated with SUD.

\section{Main text}

Methods

Animals

38 male Wistar rats, age 21 days $(50 \mathrm{~g})$ (come from the CCA-UFOP) divided randomly, into 4 groups (4 animals/box): (1) sedentary rats fed with standard diet (S-SD), $\mathrm{n}=14 ;$ (2) trained rats fed with standard diet (T-SD), $\mathrm{n}=9$; (3) sedentary rats fed with high sucrose diet (S-SUD), $\mathrm{n}=05$; and (4) trained rats fed with SUD (T-SUD), $\mathrm{n}=10$; marked on the tail; allocated in a room at a temperature of $24 \pm 2{ }^{\circ} \mathrm{C}$ and 12-h light/dark cycle (7 a.m. -7 p.m.). Body weight assessments: realized at weeks 1, 4, 5, 7, and 11. Food intake assessments: realized at weeks 1, 5, and 10. Approval of the study: by the Ethics Committee for Animal Use, UFOP (Protocol 45/2014). The experimental design scheme was shown in Additional file 2: Figure S2.

\section{Diets}

SD-commercial rodent feed in pellets (Nuvilab CR1 Quimtia ${ }^{\circledR}$ ) was administered for 12 weeks from weaning and SUD as previously published [13] by 8 weeks. After 4 weeks initial was started the exercise training program.

\section{Exercise training and evaluation of endurance capacity}

Local-collective glass tank of CCA-UFOP with warm water at $28 \pm 2{ }^{\circ} \mathrm{C}$ and $45 \mathrm{~cm}$ depth. Adaptation period: $15 \mathrm{~min}$ (first day) increased by $15 \mathrm{~min}$ each day until reaching $60 \mathrm{~min}$ (fourth day) (adapted from [14]). Maximal test: at the 4th (started training) and 12th (24 h after the last training section) weeks as proposed by others authors [15]. Fatigue point was adapted from [16]. Training: swimming for 8 weeks. The first 4 weeks didn't use a workload, but that was added at the fifth week $(60 \%$ of that obtained in maximal test) as adapted from [15].

\section{Water intake, urinary volume and water balance} measurements in $\mathbf{2 4} \mathrm{h}$

Rats were allocated in metabolic cages (Tecnoplast ${ }^{\circledR}$ SPA) during the 12-week period (CCA-UFOP); weighed
(SF-400 scale), individually housed for a period of $24 \mathrm{~h}$ with free access to water and food. Measurements of urine volume and water intake were realized. Samples of urine $1.5 \mathrm{~mL}$ were obtained, labeled, and frozen at $-20^{\circ} \mathrm{C}$. Calculation of water balance was done according to the equation:

$$
\begin{aligned}
\text { Water Balance }(m L / 24 h)= & (\text { Water Intake }[m L / 24 h] \\
& - \text { Urinary Volume }[m L / 24 h]) .
\end{aligned}
$$

\section{Euthanasia}

$48 \mathrm{~h}$ after the last $\mathrm{T}$ session. Collected materials: Adipose tissues inguinal, retroperitoneal and epididymal (IAT, RAT and EAT, respectively), blood and kidneys. Blood was centrifuged (CENTRIBIO 80-2 B centrifuge) at $3000 \mathrm{rpm}$ for $10 \mathrm{~min}$ to separate the plasma and then maintained at a temperature of $-20{ }^{\circ} \mathrm{C}$. Details: Additional file 4: Table S2.

\section{Determination of the LI}

LI was measured according to [17]. Animals' body mass and the nasoanal length were measured [17]. It was used formula below:

$$
\frac{\sqrt[3]{B M(g)}}{N L(\mathrm{~cm})}
$$

\section{Determination of the $B A I$}

BAI was measured according to [18]. The EAT, IAT, and RAT were removed and weighed (BEL precision scale), and used in the following equation:

$$
\frac{E A T \times I A T \times R A T(g)}{B W(g)} \times 100
$$

\section{Evaluation of RAT}

RAT analyse provide an assessment of the risk of developing cardiovascular diseases [19].

\section{Plasma and Urine Creatinine Concentration and Urine Protein}

Samples-plasma and urine; kit-commercially available kit (Labtest, Belo Horizonte/MG, Brazil)_by colorimetric modified Jaffé approach. Calculation of creatinine clearance $(\mathrm{CrCl})$ : 
$\operatorname{CrCl}(\mathrm{mL} / \mathrm{min})=[$ Urine Creatinine $(m g / d L) \times$ Urine Volume $(m L / 24 h)]$

$[$ Serum Creatinine $(m g / d L) \times 1440(\mathrm{~min})]$

Proteinuria was determined with the use of pyrogallol red technique using a commercially available kit (Bioclin, Belo Horizonte, Brazil), $n=38$ rats. Results were expressed in $\mathrm{mg} / \mathrm{mg}$ creatinine.

\section{Renal histology}

Collected material-right kidneys; Stage and calculation-as described by others [20, 21]. Equipment-light microscope (Leica DM5000). Analysis-Analysis and Image Processing Software Leica Qwin (Germany) [22].

\section{Data and statistical analysis}

Normality test-Kolmogorov-Smirnov. Statistical tests used-One-way or two-way analysis of variance (ANOVA) and Tukey's post-test for multiple comparisons following ANOVA. Software used-GraphPad Prism 7.0 for Windows (GraphPad Software, San Diego California USA). Data were expressed as mean \pm standard deviation of mean and differences between pairs of means were considered significant when the probability of type I error was less than $5 \%(\mathrm{p}<0.05)$. The data were analyzed blindly by the researchers involved.

\section{Results}

\section{Confirmation of the effectiveness of the exercise training} employed

SUD affected the physical performance at the 12nd week since only the T-SD group had a longer exhaustion time with an increase of $16.9 \%$ (Table 1).

\section{Effects of the exercise training on body composition}

At the 11th week, SUD caused a decrease of $23.8 \%$ in the body weight gain and an increase in the BAI (69\%), LI (10.5\%) and RAT weight (28.6\%) as shown in Additional file 1: Figure S1, panels A to C. T caused a decrease of $15.2 \%$ in body weight gain only in the T-SD (Additional file 3: Table S1) and a decrease in BAI (81\%), LI (15.8\%), and RAT weight (55.1\%) in T-SUD, decreasing only the BAI (54.9\%) and RAT weight (26.5\%) in T-SD.

\section{Effects of the exercise training on 24-h parameters hydric}

SUD caused a decrease of $42.9 \%$ the urinary volume, while and $\mathrm{T}$ restored these values to normal levels (Table 1). However, T caused an increase of $36 \%$ in water balance in the T-SD (Table 1).

\section{Effects of the exercise training on the renal biochemical parameters}

SUD increased in $22.9 \%$ the plasma creatinine (Fig. 1E) and decreased in $53.8 \%$ and $75 \%$ the urinary creatinine excretion and creatinine clearance, respectively (Fig. 1D, $\mathrm{F}$, respectively). $\mathrm{T}$ protected the kidney against SUDinduced modifications of plasma and urinary creatinine, creatinine clearance besides decreased urinary protein concentration and excretion in addition to protein/creatinine ratio.

\section{Effects of the exercise training on renal structure}

SUD increased in $31.5 \%$ in cell nuclei (Fig. 2C, third quadrant left side, and $\mathrm{G}$ ) and $\mathrm{T}$ reduced in $31.1 \%$ about this parameter.

\section{Discussion}

This study found that eight weeks of swimming physical training protected renal function and restored normal GFR values. Swimming training also contributed to prevention of the onset of a renal inflammatory process and decrease the risk of development of obesity induced by

Table 1 Exaustion times and hydrics parameters for SD and SUD rats, sedentary and trained

\begin{tabular}{|c|c|c|c|c|}
\hline & \multicolumn{2}{|l|}{ Standard diet (SD) } & \multicolumn{2}{|l|}{ Sucrose diet (SUD) } \\
\hline & Sedentary (S-SD) & Trained (T-SD) & Sedentary (S-SUD) & Trained (T-SUD) \\
\hline Initial ET (s) & $720 \pm 44(n=04)$ & $774 \pm 73(n=09)$ & $819 \pm 74(n=05)$ & $770 \pm 74(n=10)$ \\
\hline Final ET (s) & $709 \pm 53(n=04)$ & $853 \pm 92^{*}(n=09)$ & $791 \pm 47(n=05)$ & $782 \pm 73(n=10)$ \\
\hline Water intake (mL/24 h) & $29.5 \pm 5.7(n=14)$ & $33.9 \pm 4.2(n=09)$ & $29.0 \pm 2.2(n=05)$ & $30.9 \pm 1.9(n=10)$ \\
\hline Urinary volume (mL/24 h) & $14 \pm 2.4(n=14)$ & $9 \pm 3.9(n=09)$ & $8 \pm 1.4^{*}(n=05)$ & $14 \pm 5.9(n=10)$ \\
\hline Water balance (mL/24 h) & $15.8 \pm 5.9(n=14)$ & $24.6 \pm 6.4^{*}(n=09)$ & $21.1 \pm 3.5(n=05)$ & $17.3 \pm 5.5+(n=10)$ \\
\hline
\end{tabular}

Exaustion times before (Initial ET) and after (Final ET) were realized on the 4 th and $12 \mathrm{nd}$ ( $24 \mathrm{~h}$ after the last training section) weeks. It was used the parametric test, two-way ANOVA followed by Tukey's post-test; $\mathrm{p}<0.05$. * different from S-SD group. Measurements of water intake and urinary volume were realized on the metabolic cages, after $24 \mathrm{~h}$. It was used the parametric test, two-way ANOVA followed by Tukey's post-test; $\mathrm{p}<0.05 .{ }^{*}$ different of S-SD group; ${ }^{+}$different of T-SD group 

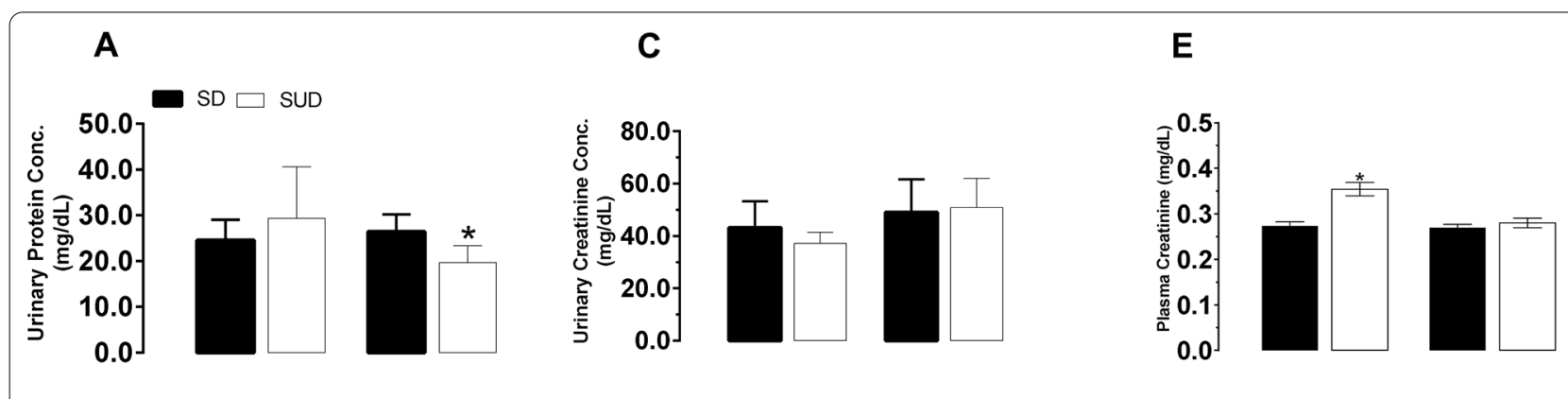

B

\section{D}

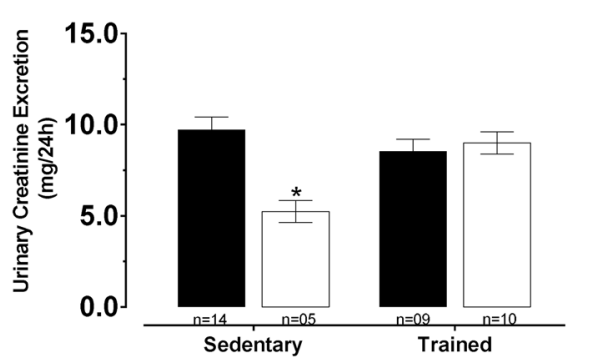

\section{$\mathbf{F}$}

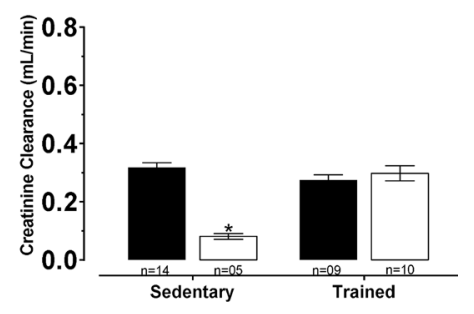

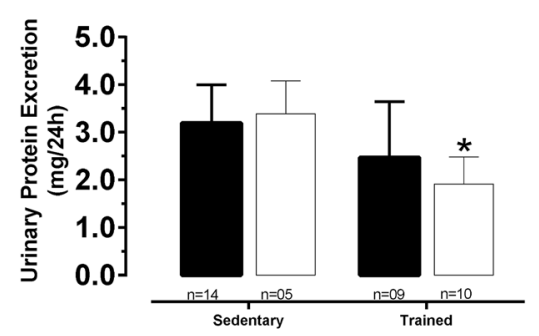

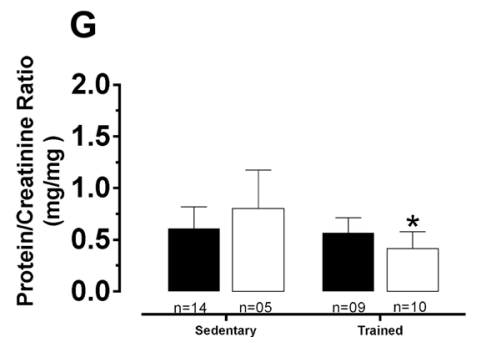

Fig. 1 Urinary protein concentration values (A), ${ }^{*}$ different from S-SUD group. Urinary protein excretion values (B), * different from S-SD and S-SUD groups. Urinary creatinine concentration values $(\mathbf{C})$, there was no difference between groups. Urinary creatinine excretion values $(\mathbf{D})$, *different from S-SD, T-SD and T-SUD groups. Plasma creatinine (E), ${ }^{*}$ different from S-SD, T-SD and T-SUD groups. Creatinine clearance values $(\mathbf{F}),{ }^{*}$ different from S-SD, T-SD and T-SUD groups. Protein/Creatinine ratio values $(\mathbf{G}),{ }^{*}$ different from S-SUD group. SD (Standard Diet), SUD (Sucrose Diet) and $\mathrm{T}$ (swimming training). All measurements were realized after 8th week of T. It was used the parametric test, two-way ANOVA followed by Tukey's post-test; $p<0.05$. The bars represent group mean data. Differences among the pairs of means are indicated by different signs

SUD since the body composition parameters (BAI, LI, and RAT weight) were lower in trained group.

Although some authors have not observed impairment in the physical performance of animals fed a diet rich in simple carbohydrates [23], SUD contributed negatively to the physical performance of the animals in our study. After 12 weeks of swimming exercise training, the total time to exhaustion was higher only in animals of the T-SD group.

Sugar seems to increase parameters, such as BAI $[18,24-26]$, LI [26], and RAT weight $[27,28]$ that contribute to the development of cardiovascular diseases [25]. In our study we observed that all these parameters were increased by SUD. Our results show that the exercise reduced BAI in the T-SUD (81\%) and T-SD (54.9\%) groups and LI and RAT weight in the T-SUD group although some studies haven't shown the effects of physical exercise in reducing the BAI, LI and RAT weight [13,
19, 24, 29] promoted by high sugar content. A possible explanation for these exercise-associated effects is energetic homeostasis tends to been maintained in mammals through a balance between energy intake and expenditure, thus influencing body weight [30] doing the $\mathrm{T}$ have an important role in controlling these factor [31, 32].

Some studies have shown that a sugar intake can have influence (or not) in parameters like water intake [8, 33, 34], urinary volume [8], and water balance [8] in rodents. In this study, SUD didn't affect water intake in any group and decreased urinary volume, but the exercise training restored to normal levels. The T-SD group had a higher water balance, demonstrating that swimming promotes changes in the hydric balance depending on the diet to which it was assigned. The T-SD group seems to have had a higher expenditure of water by other means than urinary excretion. Therefore, when swimming was undertaken by the SUD group, these differences don't appear. 


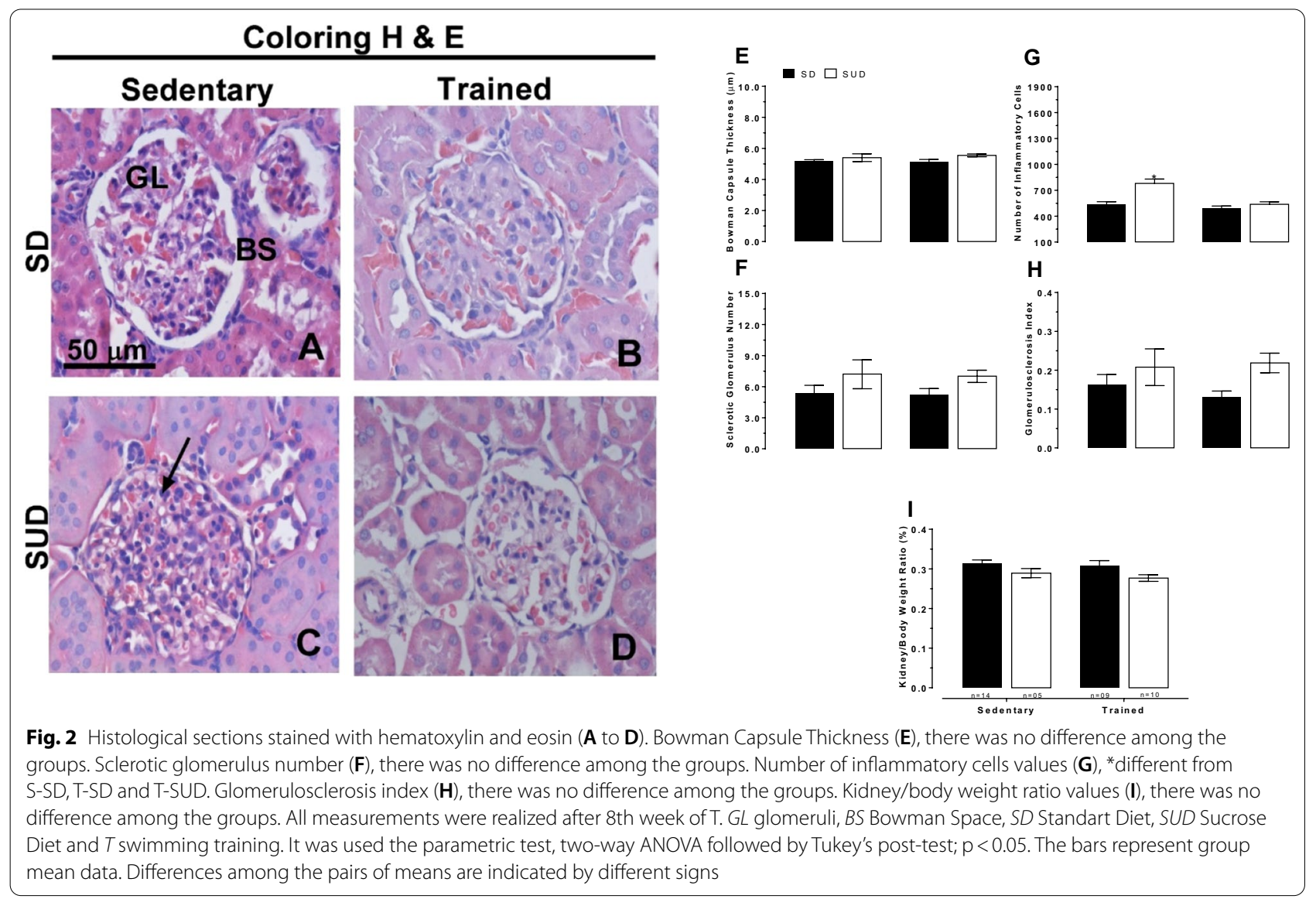

There are no studies that demonstrate how changes in water balance occur in response to sugar overload in rodents and outline the mechanisms by which this process happens. Studies that investigate the hydric parameters are necessary to clarify many of the physiological changes that may occur in rodents fed with sugar overload, since these issues may be related to some compensation of the physiological system to maintain homeostasis, mainly of the renal system.

It is known that the overload sugar can modifying the following parameters: (1) proteinuria [34, 35], (2) plasma and urinary creatinine [34], and (3) creatinine clearance (used as estimate of the GFR) [34, 36]. In our study, SUD increased plasma creatinine and decreased urinary creatinine excretion and creatinine clearance. $\mathrm{T}$ led to a reversal in GFR alterations by bringing this parameter back to normal levels corroborating with others [7]. In addition, the $\mathrm{T}$ decreased urinary protein concentration and excretion and in protein/creatinine ratio.

Sugar has also been seen as a villain that can cause renal inflammation, as noted in other study [37]. In our research, SUD caused an increase in cell nuclei suggesting an increase in the number inflammatory infiltrates. T protected against these SUD-induced changes (decreasing this parameter in $31.1 \%$ ), thus corroborating the results presented by others authors [38]. All of the other parameters weren't affected neither by SUD nor for T (Fig. 2E, F, H, and I).

It was observed that damage to kidney function appeared earlier than structural renal damage in response to excess sugar in the SUD. High glucose-induced oncotic pressure alterations didn't initially modify the structure of the glomerulus but did modify renal function probably by increasing the excretion of urinary proteins, thus increasing proteinuria and decreasing GRF [39]. In addition, the time of exposure to the diet and age of animals (young animals) could have provided protection for the renal structure conferred by mechanisms of adaptation to sugar overload.

Collectively, our study demonstrated that $\mathrm{T}$ prevented SUD-induced impairment of kidney functional parameters and was efficient in preventing an SUD-induced increase adipose tissue deposits. 


\section{Limitations}

We lacked investigate the cardiovascular parameters complementing the data aiming to observe the effects of sugar overload also on these parameters, being able to make a direct relationship between renal parameters, body composition and cardiovascular parameters in response to SUD and T.

\author{
Abbreviations \\ UFOP: Federal University of Ouro Preto; T: Swimming training; SUD: High \\ sucrose diet; S-SD: Sedentary standard diet; S-SUD: Sedentary SUD; T-SD: \\ Trained standard diet; T-SUD: Trained SUD; BAI: Body adiposity index; LI: Lee \\ index; RAT: Retroperitoneal adipose tissue; EAT: Epididymal adipose tissue; IAT: \\ Inguinal adipose tissue; RAT: Retroperitoneal adipose tissue; $\mathrm{CrCl}$ : Creatinine \\ clearance; GFR: Glomerular filtration rate; CCA-UFOP: Center of Animal Science \\ of UFOP.
}

\section{Supplementary Information}

The online version contains supplementary material available at https://doi. org/10.1186/s13104-021-05790-7.

Additional file 1: Figure S1. Body adiposity index values (panel A), ${ }^{*}$ different from S-SD, T-SD and T-SUD groups, ${ }^{+}$different from S-SD group. Lee index values (panel B), * different from S-SD, T-SD, T-SUD groups. Retroperitoneal adipose tissue weight values (panel C), ${ }^{*}$ different from S-SD, T-SD and T-SUD groups, ${ }^{* *}$ different from S-SD group. It was used the parametric test, two-way ANOVA followed by Tukey's post-test; $p<0.05$; all the measurements above were realized after the $8^{\text {th }}$ week of T. SD (Standart Diet), SUD (Sucrose Diet) and T (swimming training). The bars represent group mean data. Differences among the pairs of means are indicated by different signs.

Additional file 2: Figure S2. Scheme of experimental design showing the different stages for the development of the model, allocation of animals in different groups and main experimental tests carried out in this study. Weaned rats from CCA-UFOP were fed diets containing 68\% carbohydrates, 33\% standard commercial feed, 33\% condensed milk, 7\% sucrose and the remaining water or standard pellet diet for 12 weeks since weaning. In the 4 th week, the rats were adapted (4 days) to the aquatic environment and the animals related to the trained groups underwent swimming training for 8 weeks. The exhaustion time protocol was performed at weeks 4 and 12 to assess the animals' physical performance. The measurements of water intake and urinary volume performed in a metabolic cage were performed on the 12nd week, in addition to the renal (for analysis of renal function and histology) and body composition (BAI, LI and RAT weight) experiments, which were performed $48 \mathrm{~h}$ after last $T$ session at the end of the 12 nd week, at which time the rats were euthanized and samples of blood and kidney tissue were collected for analysis. Body weight measurements were taken at weeks 1, 4, 5, 7 and 11 and food intake measurements were taken at weeks 1, 5 and 10. CCAUFOP (Center of Animal Science of UFOP); SD (standart diet); SUD (sucrose diet); $T$ (swimming training).

Additional file 3: Table S1. Body weight measurements and food intake before and after $\mathrm{T}$.

Additional file 4: Table S2. Table with additional information about the study.

\section{Acknowledgements}

The authors thank the support by Center of CCA-UFOP, Morphopathology Laboratory and NUPEB/UFOP Advanced Microscopy and Microanalysis Multiuser Laboratory.

\section{Information regarding}

Criteria for the inclusion of animals in the study; Criteria for the exclusion of animals in the study; Animals excluded of the study; Handling of the animals; Monitoring of the animals; Number of animals per experimental procedures; Justification of the choice of experimental procedures; Sample calculation; Euthanasia, were described in Additional file 4: Table S2.

\section{Authors' contributions}

JAS, ABGP: Design of experimental protocols; performed the experiments; analyzed data; interpret results of experiments; prepared figures; drafted manuscript. ECO, DBC, NLT, WGL: edited and revised manuscript. NLT, WGL: Performed histology. NLT, LKB: helped in experiments. LKB: Conception and design the research; analyzed data; helped in experiments; interpret results of experiments; prepared figures; drafted manuscript; edited and revised manuscript. All authors read and approved final manuscript.

\section{Funding}

This study was supported by Minas Gerais State Research Foundation (FAPEMIG) and the National Council for Scientific and Technological Development $(\mathrm{CNPq})$ with financial support for conducting the research.

\section{Availability of data and materials}

The datasets used and/or analyzed during the current study are available from the corresponding author on reasonable request.

\section{Declarations}

\section{Ethics approval and consent to participate}

This study approval by the Ethics Committee for Animal Use-UFOP (Protocol 45/2014). There was approval of the use of the Wistar rat strain and the number of animals (previously obtained from sample calculation) and to experiments that were carried out. Consent was granted by the animal research ethics committee via written document.

\section{Consent for publication}

Not applicable.

\section{Competing interests}

The authors declare that they have no competing interests.

\section{Author details}

${ }^{1}$ Physical Education School, Federal University of Ouro Preto (UFOP), Ouro Preto, MG 35,400-000, Brazil. ${ }^{2}$ Dept. of Biological Sciences-ICEB, UFOP, Ouro Preto, MG, Brazil. ${ }^{3}$ Dept. of Pharmacology, ICB, Federal University of Minas Gerais, Belo Horizonte, MG, Brazil.

Received: 8 March 2021 Accepted: 15 September 2021

Published online: 26 September 2021

\section{References}

1. Johnson RJ, et al. Potential role of sugar (fructose) in the epidemic of hypertension, obesity and the metabolic syndrome, diabetes, kidney disease, and cardiovascular disease. Am J Clin Nutr. 2007;86(4):899-906.

2. Gross LS, et al. Increased consumption of refined carbohydrates and the epidemic of type 2 diabetes in the United States: an ecologic assessment. Am J Clin Nutr. 2004;79(5):774-9.

3. Neilson EG. The fructose. Nation. 2007;18(10):2619-21.

4. MalikVS, et al. Sugar-sweetened beverages and risk of metabolic syndrome and type 2 diabetes: a meta-analysis. Diabetes Care. 2010;33(11):2477-83.

5. Roberts $C K$, et al. Oxidative stress and dysregulation of $\mathrm{NAD}(\mathrm{P}) \mathrm{H}$ oxidase and antioxidant enzymes in diet-induced metabolic syndrome. Metabolism. 2006;55(7):928-34.

6. Velasquez MT, et al. Diabetic glomerulopathy in the SHR/N-corpulent rat: role of dietary carbohydrate in a model of NIDDM. Diabetologia. 1995;38(1):31-8. 
7. $\mathrm{Hu} \mathrm{G}$, et al. Chronic exercise provides renal-protective effects with upregulation of fatty acid oxidation in the kidney of high fructose-fed rats. Am J Physiol Renal Physiol. 2020;318(3):F826-34.

8. Muller $\mathrm{CR}$, et al. Aerobic exercise training prevents kidney lipid deposition in mice fed a cafeteria diet. Life Sci. 2018;211:140-6.

9. Preuss $H$, et al. Sugar-induced blood pressure elevations over the lifespan of three substrains of wistar rats. J Am Coll Nutr. 1998;17:36-47.

10. Gluckman PD, Hanson MA, Beedle AS. Non-genomic transgenerational inheritance of disease risk. BioEssays. 2007:29(2):145-54

11. Inadera H. Developmental origins of obesity and type 2 diabetes: molecular aspects and role of chemicals. Environ Health Prev Med. 2013;18(3):185-97.

12. Bremer AA, Mietus-Snyder M, Lustig RH. Toward a unifying hypothesis of metabolic syndrome. Pediatrics. 2012;129(3):557-70.

13. Barbosa de Queiroz K, et al. Physical activity prevents alterations in mitochondrial ultrastructure and glucometabolic parameters in a high-sugar diet model. PLoS ONE. 2017;12(2):e0172103.

14. Seo TB, et al. Involvement of Cdc2 in axonal regeneration enhanced by exercise training in rats. Med Sci Sports Exerc. 2006;38(7):1267-76.

15. Botezelli JD, et al. Exercise counteracts fatty liver disease in rats fed on fructose-rich diet. Lipids Health Dis. 2010;9:116.

16. Casimiro-Lopes $\mathrm{G}$, et al. Maternal prolactin inhibition during lactation affects physical performance evaluated by acute exhaustive swimming exercise in adult rat offspring. Horm Metab Res. 2012:44(2):123-9.

17. Bernardis LL, Patterson BD. Correlation between "Lee index" and carcass fat content in weanling and adult female rats with hypothalamic lesions. J Endocrinol. 1968;40(4):527-8.

18. Oliveira MC, et al. Acute and sustained inflammation and metabolic dysfunction induced by high refined carbohydrate-containing diet in mice. Obesity (Silver Spring). 2013;21(9):E396-406.

19. De Queiroz KB, et al. Endurance training increases leptin expression in the retroperitoneal adipose tissue of rats fed with a high-sugar diet. Lipids. 2014;49(1):85-96.

20. Totou NL, et al. Swimming exercise demonstrates advantages over running exercise in reducing proteinuria and glomerulosclerosis in spontaneously hypertensive rats. Physiol Int. 2018;105(1):76-85.

21. Souza JA, et al. Swimming training improves cardiovascular autonomic dysfunctions and prevents renal damage in rats fed a high-sodium diet from weaning. Exp Physiol. 2020;106:412-26.

22. Molinaro EM, Caputo LFG, Amendoeira MRR. Concepts and methods for training professionals in health laboratories. In: Organization of Etelcia Moraes Molinaro, Luzia Fátima Gonçalves Caputo e Maria Regina Reis Amendoeira, vol. 2. Rio de Janeiro: EPSJV; IOC; 2010. p. 290. ISBN: 978-85-98768-41-0.

23. Sam Lee J, et al. Interaction of diet and training on endurance performance in rats. Exp Physiol. 2001;86(4):499-508.

24. De Queiroz KB, et al. Endurance training blocks uncoupling protein 1 up-regulation in brown adipose tissue while increasing uncoupling protein 3 in the muscle tissue of rats fed with a high-sugar diet. Nutr Res. 2012;32(9):709-17.

25. de Queiroz KB, et al. Molecular mechanism driving retroperitoneal adipocyte hypertrophy and hyperplasia in response to a high-sugar diet. Mol Nutr Food Res. 2014;58(12):2331-41.

26. Oliveira DTD, et al. High-sugar diet leads to obesity and metabolic diseases in ad libitum-fed rats irrespective of caloric intake. Arch Endocrinol Metab. 2020;64(1):71-81.

27. Malafaia $A B$, et al. Indução de obesidade com sacarose em ratos. $A B C D$ Arquivos Brasileiros de Cirurgia Digestiva (São Paulo). 2013;26(suppl 1):17-21.

28. Castellanos Jankiewicz AK, et al. Adipose tissue redistribution caused by an early consumption of a high sucrose diet in a rat model. Nutr Hosp. 2015;31:2546-53. https://doi.org/10.3305/nh.2015.31.6.8935.

29. Carmo $M$, et al. Swimming exercise did not ameliorate the adverse effects of high-sugar diet in young rats. J Exer Physiologyonline. 2017;20:177-83.

30. Borer KT. Exercise endocrinology, 2nd edn. University of Michigan, Human Kinetics, 2003. p. 273.

31. Wing RR, Hill JO. Successful weight loss maintenance. Annu Rev Nutr. 2001;21(1):323-41.

32. Zhang N, Bi S. Effects of physical exercise on food intake and body weight: role of dorsomedial hypothalamic signaling. Physiol Behav. 2018;192:59-63.

33. Preuss $\mathrm{HG}$, et al. Effects of high sugar diets on renal fluid, electrolyte and mineral handling in rats: relationship to blood pressure. J Am Coll Nutr. 1994;13(1):73-82

34. Francisqueti F, et al. Effect of Gamma-Oryzanol as therapeutic agent to prevent cardiorenal metabolic syndrome in animals submitted to high sugar-fat diet. Nutrients. 2017;9(12):1299.

35. El-Bassossy HM, Shaltout HA. Allopurinol alleviates hypertension and proteinuria in high fructose, high salt and high fat induced model of metabolic syndrome. Transl Res. 2015;165(5):621-30.

36. Roysommuti $\mathrm{S}$, et al. Excess dietary glucose alters renal function before increasing arterial pressure and inducing insulin resistance. Am J Hypertens. 2002;15(9):773-9.

37. Fan C-Y, et al. Betaine supplementation protects against high-fructoseinduced renal injury in rats. J Nutr Biochem. 2014;25(3):353-62.

38. Cao G, et al. Beneficial effect of moderate exercise in kidney of rat after chronic consumption of cola drinks. PLOS ONE. 2016;11(3):e0152461.

39. Guyton AC. Textbook of medical physiology. Hoboken: Elsevier; 2006. p. $181-94$.

\section{Publisher's Note}

Springer Nature remains neutral with regard to jurisdictional claims in published maps and institutional affiliations.
Ready to submit your research? Choose BMC and benefit from:

- fast, convenient online submission

- thorough peer review by experienced researchers in your field

- rapid publication on acceptance

- support for research data, including large and complex data types

- gold Open Access which fosters wider collaboration and increased citations

- maximum visibility for your research: over $100 \mathrm{M}$ website views per year

At BMC, research is always in progress.

Learn more biomedcentral.com/submissions 